\title{
Surface resistance of ESD-protected worksurfaces- measurement, modelling and estimation considerations
}

\author{
Zsolt Kemény ${ }^{1}$, Zsolt János Viharos ${ }^{1,2}$, Krisztián Balázs Kis ${ }^{1}$, Róbert Csontos ${ }^{3}$, Tamás Kovács ${ }^{3}$, Kornél \\ Németh $^{3}$ \\ ${ }^{1}$ Institute for Computer Science and Control, Hungarian Academy of Sciences, Kende u. 13-17., H-1111 Budapest, Hungary \\ ${ }^{2}$ Pallasz Athéné University, Izsáki út 10., H-6000 Kecskemét, Hungary \\ ${ }^{3}$ iQor Global Services Hungary Kft., Vásártér u. 1., H-9700, Szombathely, Hungary
}

\begin{abstract}
The prevention of electrostatic discharge (ESD) is of crucial importance in the electronics industry, and surfaces of workstations have to be of specific resistance for effective ESD protection. The paper presents results of an R\&D project which investigated the-so far rarely researched-dependence of worksurface resistance on ambient conditions and surface contamination in a live industrial environment. Upon examination of known and assumed dependencies, measurement and instrumentation are outlined, relying on existing automated facility management, autonomous devices, and manual measurement/logging. Further parts of the paper report on an analysis of the data obtained, as well as their use in building models of surface resistance, employing feature selection metaheuristics applied in combination with artificial neural networks. Surface resistance models built with approximately one year's worth of measurement data yielded estimations with $12 \%$ mean relative error, and showed that surface resistance can be estimated relying on data that can be obtained by contactless and remote measurement, without direct interference with work processes.
\end{abstract}

\section{Section: RESEARCH PAPER}

Keywords: manufacturing; ESD protection; surface resistance; modelling

Citation: Zsolt Kemény, Zsolt János Viharos, Krisztián Balázs Kis, Róbert Csontos, Tamás Kovács, Kornél Németh, Surface resistance of ESD-protected worksurfaces-measurement, modelling and estimation considerations, Acta IMEKO, vol. 6, no. 4, article 2, December 2017, identifier: IMEKO-ACTA-06 (2017)-04-02

Section Editor: Alexandru Salceanu, Technical University of lasi, Romania

Received April 26, 2017; In final form July 31, 2017; Published December 2017

Copyright: @ 2017 IMEKO. This is an open-access article distributed under the terms of the Creative Commons Attribution 3.0 License, which permits unrestricted use, distribution, and reproduction in any medium, provided the original author and source are credited

Funding: Work presented here has been supported by the grants of the Highly Industrialised Region in Western Hungary with limited R\&D capacity: "Strengthening of the regional research competencies related to future-oriented manufacturing technologies and products of strategic industries by a research and development program carried out in comprehensive collaboration", under grant No. VKSZ_12-1-2013-0038

Corresponding author: Zsolt Kemény, e-mail: zsolt.kemeny@sztaki.mta.hu

\section{INTRODUCTION}

Research and development of the past 1 to 2 decades brought forth data processing and model building tools that are able to tackle the complex interdependencies of large production systems at multiple levels of organizational and functional hierarchy, as well as sophisticated methods and technologies for prediction, planning and control of industrial processes. Several of these have ripened from experimental pilot to industrial application, and find growing acceptance in production environments that are otherwise pressed by tightening environmental and health regulations, and by increasing competition that requires costs to be cut while maintaining or improving product quality, flexibility and responsiveness. An important development contributing to these trends is the increase of process transparency by means of massive unique identification, process/product data and measured quantities, allowing better models to be built and utilized, possibly also yielding a more accurate picture of the borders of safe operating conditions. The latter can, in turn, be approached more closely, resulting in savings and improved quality and process safety guarantees.

The specific case examined in the paper is that of the electronics industry where products must be protected from electrostatic discharge (ESD), especially at stages of production, 
maintenance, or repair where no protective shielding of the product is present. ESD occurs when electrostatic charges accumulated in production equipment, clothing of personnel, etc., are discharged in an ESD event. Discharge passing through semiconductor components may inflict irreparable damage which can remain hidden long enough for a damaged device to slip through immediate quality checks - such risks must, therefore, be removed from the processes of production and handling. This consists in ensuring that (1) electrostatic charges accumulate as little as possible in the environment, equipment, and personnel, and (2) if a discharge event does occur after all, discharge current must be limited to protect sensitive components from overcurrent. In industrial practice, this is ensured by (1) the use of conductive materials for floors, clothing, worksurfaces (Figure 1) and certain tools, as well as protective ground connections at specific points of production equipment, and by (2) the surface resistivity of materials in possible physical contact with the semiconductor components being within a range that allows draining of accumulated charge but keeps discharge current within safe limits [1], [2].

The transfer resistance of surfaces depends on several ambient conditions as well as deposits on the surface - in present-day practice, neither precise and frequent measurement of the relevant conditions, nor a minimal-impact acquisition of actual resistance values are part of industrial practice. While contactless methods are known for the measurement of charge dissipation properties [3], their setup would still interfere with the work processes. Therefore, an accurate model of the dependence of surface resistance on ambient conditions and process parameters is not relied on in present-day industrial practice, implying relatively rough estimations and wide safety margins that are maintained at high costs. It is expected that more accurate knowledge of a surface resistance model will eventually contribute to improved efficiency in maintaining safe operating conditions. Nonetheless, data acquisition and model building are only of practical relevance to the industry if they can be rolled out and robustly deliver added value in a true industrial environment where interdependencies are complex and largely unknown, and data acquisition can be limited and never has top priority.

Extending a previous IMEKO TC4 conference publication [4], the paper presents a measurement instrumentation, data pre-processing, and model acquisition setup in the context of an R\&D project that has collected measurement data of ambient conditions and work activity logs assumed to be relevant for modelling the surface resistance of worksurfaces of manually operated ESD-protected workstations. The key objective of the work presented here is to examine the viability of the aforementioned data acquisition and modelling as a field-deployable method, and much less the establishment of an accurate

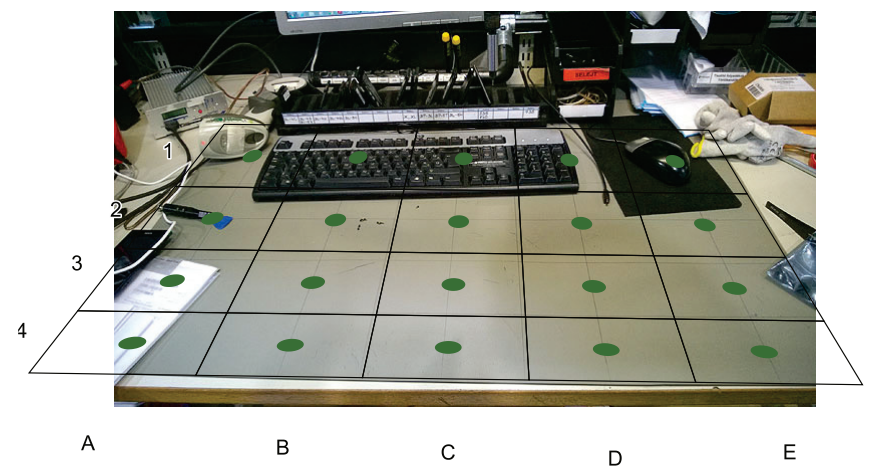

Figure 1. Example of an ESD-protected worksurface. Green marks show the locations of resistance measurement carried out in this research. model of one or several specific ESD-protective materials at any cost. In further parts, the paper is structured as follows. After an overview of preliminaries (Section 2), the extent and methods of measurement are presented (Section 3), followed by first findings of raw data (Section 4), and the concept of data preparation, model building, and results of modelling itself (Section 5). Section 6 recapitulates the novelties achieved by the research so far, and highlights further possibilities of measurement and online diagnostics.

\section{PREVIOUS WORK}

\subsection{ESD protection in literature}

The mainstream of ESD-related literature deals, in fact, not with ESD protection directly but with the nature and effects of ESD events, assuming that discharge does already occur [5], [6]. A major share is taken by models (i.e., substituting circuits) of equipment or personnel potentially carrying accumulated charge [7], [8], facilitating comparative characterization [5], formal analysis, simulation of ESD events [6], and definition of robustness requirements for semiconductor components and their protective circuits.

The second major group of works deals with robustness of semiconductors, devices and protective circuits against electrostatic discharge [9]-[11]. Also here, the occurrence of an ESD event is assumed, while research presented here is aimed at ensuring their continuous prevention - hence, little of these two major problem areas are directly related to our focal problem.

A third - much narrower - segment, more relevant to the topic of the paper, focuses on materials suitable for ESD protection of work environment, tools, clothing, and packaging [1], [12]-[15]. While these give valuable guidance for expected dissipative characteristics, only a fraction of the sources deals in detail with the influence of certain ambient conditions on material properties [1], [16]-[18].

While the thin corpus of existing literature in the third area made first steps of the presented research difficult, it still allowed one to recognise that the current project, due to its specific perspective, would have to cope with two requirements without significant preliminaries at hand. So far, existing literature has, namely, not aimed for (1) acquisition of material properties under conditions of live production processes (i.e., measurements known so far assumed a laboratory environment where conditions can be manipulated as desired and measurements can be taken without having to avoid interference with work processes), and (2) building a model that covers possible dynamics of ambient conditions (some of them partially or poorly measurable) and can be queried in real-time for early warnings or recommended cleaning, adjustment, etc. of ESDprotected workstations.

\subsection{Relevant conditions in other domains}

Earlier experience has already revealed that dust settling on the worksurface, in combination with humidity and temperature of ambient air, has impact on the resistance of ESD-protected worksurfaces. Therefore, it is worth examining how these conditions are represented in literature in other domains [19], [20]. Relevant in this context are results regarding particulate matter, aerosols and settling of dust [21]-[23] which reveal much regarding expected fluctuations of dust density (even though, care must be taken regarding the specific composition and ratio of mineral particles, cellulose and skin fragments 
which differ in outdoor environments and closed airspaces of manufacturing facilities).

While some sources deal with the mechanical behaviour and handling of dust (e.g., accumulation and removal from photovoltaic panels [24]), others have investigated the conductive properties of dust and moisture, with particular attention to their effect on dielectrics such as high voltage isolators [21] and printed circuit boards (PCBs) [19], [26].

Research has also been extended to transmittance and reflectance of dust-contaminated surfaces, especially in the infrared spectrum. A number of sources point out that moisture captured by settled dust exhibits definite spectral patterns [27] which are potentially useful in estimating surface resistance properties as well - the more so as this would allow online contactless and remote measurement with minimal impact on ongoing work processes.

\subsection{Industrial experience}

Empirical experience has shown over the past few decades that ambient temperature, humidity and deposits on the surface have impact on the resistance of ESD-protected worksurfaces - nonetheless, it must be noted that these are much influenced by production practice, such as cleaning, choice of materials in tools and clothing, and artificial control of ambient conditions. The Ishikawa diagram shown in Figure 2 reflects the relevance of contributing factors recognized in today's production practice. Note that the relevance predicates shown reflect the impact of factors under nominal operating conditions which are kept in safe distance from potential risk zones by a wide margin that precludes hazards, even under limited opportunities of measurement and intervention. Regarding relative humidity, a $30 \%$ limit is seen as a rule of thumb: below this value the resistance of rubber, and most polymer, surfaces may rise beyond safe limits, necessitating very costly humidity control, e.g., in cold and dry outdoor weather [16]-[18].

\section{MEASUREMENT SETUP: CONCEPT AND EXTENT}

\subsection{Purpose of measurement}

As outlined before, the purpose of measurements presented here is to gain more accurate knowledge of the dependence of the surface resistance of ESD-protected worksurfaces on selected ambient conditions in a live industrial environment (temperature, relative humidity, floating/settled dust, regular work-related activities and cleaning/maintenance measures). The quantities of interest are shown in an Ishikawa diagram revised in the course of our research (Figure 2). The figure shows a shift of attention towards quantities that had less impact under close-to-nominal operating conditions (see the framed area at the bottom left of the diagram).

Due to the nature of the research presented here, measurements had to be carried out in live industrial environment where (1) ambient conditions cannot be varied at will as one would do in a laboratory or test pad (nonetheless, the measurements will span the operating area in ambient conditions that nominal work processes usually cover), and (2) ongoing work processes and hard requirements on health, safety and product quality guarantees do restrict the quantities to be measured, and the ways, places and timing at which they can be measured (e.g., measurement directly at the worksurface is only possible after completion of a shift, with all products stowed safely, and personnel not present). While these conditions do limit the possibilities of model acquisition, similar circumstances have to be expected in an industrial roll-out as a "retrofit" in existing and operating facilities.

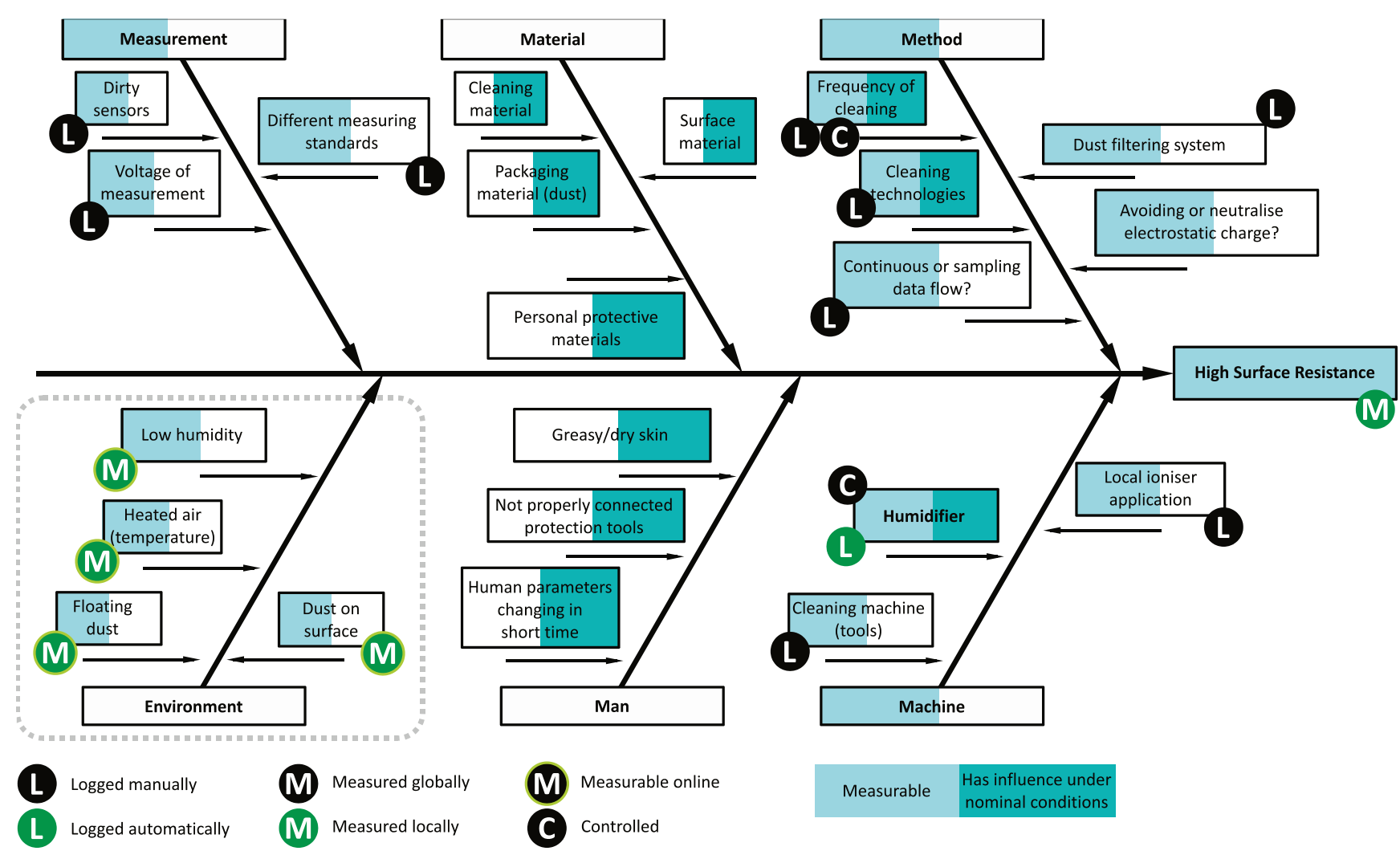

Figure 2. Revised diagram of dependencies and controllable/measurable quantities - note the shift of focus towards previously marginal factors (see circled are at the bottom left of the fishbone diagram). 


\subsection{Measurement and instrumentation}

Data were gathered in a constantly operating facility under normal operating conditions, from three groups of sources:

(1) Downloads from automated facility management records covered outdoor and indoor facility-level temperature, fan-air temperature, and relative humidity values. These quantities were measured, as part of the normal functioning of the rooftop HVAC (heating, ventilation, air conditioning) units at ceiling height. Two rooftop HVAC units were selected as source (marked as RT4 and RT6), delivering 650-700 datasets of 8 scalar values weekly (sampling every 15 minutes).

(2) Independent logging devices delivering data via periodic manual downloads were installed on a support pillar in the vicinity (within less than $2 \mathrm{~m}$ ) of the workstation, approximately at the height of the ESD-protected worksurface of the station in question. The locations of the workstation and the logging devices were selected to be near the path of frequent logistics processes inside the facility (typically transporting cardboard boxes on carts), allowing any possible short-time effect of material handling to show in the dust density measurements. Logging devices were designed and procured in-house, and contain a set of sensors, an independently running real-time clock (RTC), and a microcontroller for immediate conversion, time-stamped storage (EEPROM) and communication of measurements through a periodically connected serial interface (see Figure 3). Logging devices have their own independent power source. Relative humidity, wet-bulb and ambient temperature measurements relied on off-the-shelf semiconductor components which communicate measured values digitally to the microcontroller. Floating dust density was likewise measured using a commercial sensor comprising a sampling channel with regulated temperature difference ensuring a steady air stream, and an optical sensor measuring the transmissivity of sampled air [28]. The logging devices yielded 1900-2100×3 scalars a week for relative humidity, wet-bulb and ambient

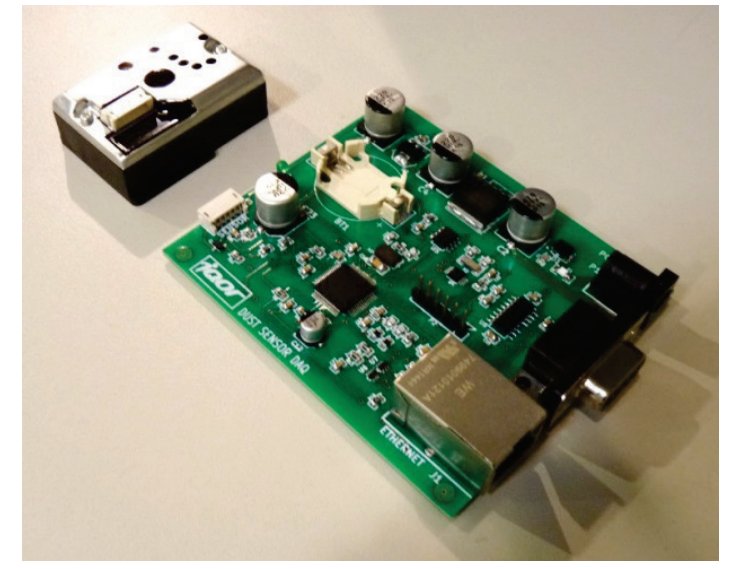

Figure 3. Example of logging device (bottom right) and dust density sensor (top left).

temperature, and 3800-4000 scalars a week for floating dust density.

(3) Manual measurements were carried out once a week, after the first or second shift of the day. During these measurements, ambient air temperature and relative humidity were measured by a handheld device directly above the worksurface, while manually measured resistance values were taken between a common ground point (accessible as a metallic contact at the workstation) and 20 discrete grid points of the worksurface of the selected workstation (Figure 1). The latter measurements were carried out with a handheld instrument [29], connected to a cylindrical probe of standard weight and geometry as specified in [30]. Manual measurements thus delivered $20+2$ scalars a week. In addition, cleaning event dates and times were logged whenever the worksurface was cleaned (1-5 times a week).

Table 1 gives a summary of the quantities measured in the data collection period, as well as their spatial range and method

Table 1. Summary of quantities measured in the data collection period.

\begin{tabular}{|c|c|}
\hline $\begin{array}{l}\text { Spatial range / measured quantities } \\
\text { (data source) }\end{array}$ & $\begin{array}{l}\text { Frequency of measurements } \\
\text { (typical amount of data per week) }\end{array}$ \\
\hline $\begin{array}{l}\text { Entire facility covered by } 2 \text { points of measurement } \\
\text { - Relative humidity } \\
\text { - Indoor temperature } \\
\text { - Fan air temperature } \\
\text { - Outdoor temperature } \\
\text { (Logged automatically by rooftop HVAC units) }\end{array}$ & Every 15 minutes (650-700×8 scalars) \\
\hline $\begin{array}{l}\text { Vicinity of workstation } \\
\text { - Density of floating dust } \\
\text { (Logged automatically by dedicated device) }\end{array}$ & Every 3 minutes (3800-4000×1 scalar) \\
\hline $\begin{array}{l}\text { Vicinity of workstation } \\
\text { - Relative humidity } \\
\text { - Ambient temperature } \\
\text { - Wet bulb temperature } \\
\text { (Logged automatically by dedicated device) }\end{array}$ & Every 5 minutes (1900-2100×3 scalars) \\
\hline $\begin{array}{l}\text { Directly at workstation } \\
\text { - Surface resistance at } 20 \text { pre-defined points on ESD-protected worksurface mat } \\
\text { - Relative humidity } \\
\text { - Ambient temperature } \\
\text { (Measured and logged manually) }\end{array}$ & Ca. $1 \times$ per week ( $20+2$ scalars) \\
\hline $\begin{array}{l}\text { Directly at workstation } \\
\text { - Worksurface cleaning event } \\
\text { (Logged manually) }\end{array}$ & Up to $5 \times$ per week (discrete events) \\
\hline
\end{tabular}


of measurement and registration.

Clearly, the selection of measured quantities, and the degree of measurement automation leaves much reserve to be exploited for successful roll-out in everyday production measuring the surface resistance presents by far the tightest bottle-neck here, due to its labour-intensive nature and high uncertainty. Some limitations of instrumentation and measurement were set by the extent of this particular project (budget and workforce limits, in particular), forcing some key approaches, such as infrared spectrometry, to be postponed, while other constraints were set by the production environment (e.g., resistance measurements are confined to time slots between shifts). Some quantities deemed relevant in the Ishikawa diagram cannot be measured with sufficient certainty. Wherever possible, we strove to either balance out such uncertainties by measuring across an entire spectrum of conditions (e.g., staff rotation reduces fluctuation due to individual differences in typical skin resistance, skin flaking, etc.), or by keeping influencing factors constant (e.g., fixed types of operations carried out at the selected workstation).

\section{EVALUATION OF RAW DATA}

Measurements were taken on a regular basis — with minor interruptions for operational reasons - from calendar week 35 of 2015 until week 34 of 2016, yielding ca. 700,000 scalar values. A first examination of raw data did already confirm consistency of values of the same quantity measured by different sensors, and reveal simple relations. Neither indoor temperature nor relative humidity showed much variation across the factory airspace, suggesting that a facility-wide rollout is likely to succeed with relatively few temperature and humidity measuring locations. The impact of the difference of indoor and outdoor temperature on indoor relative humidity is clearly recognizable, as is the effect of relative humidity on surface resistance which begins to rise at values below $30 \%$ (see Figure 4), both findings confirming previous industrial experience.
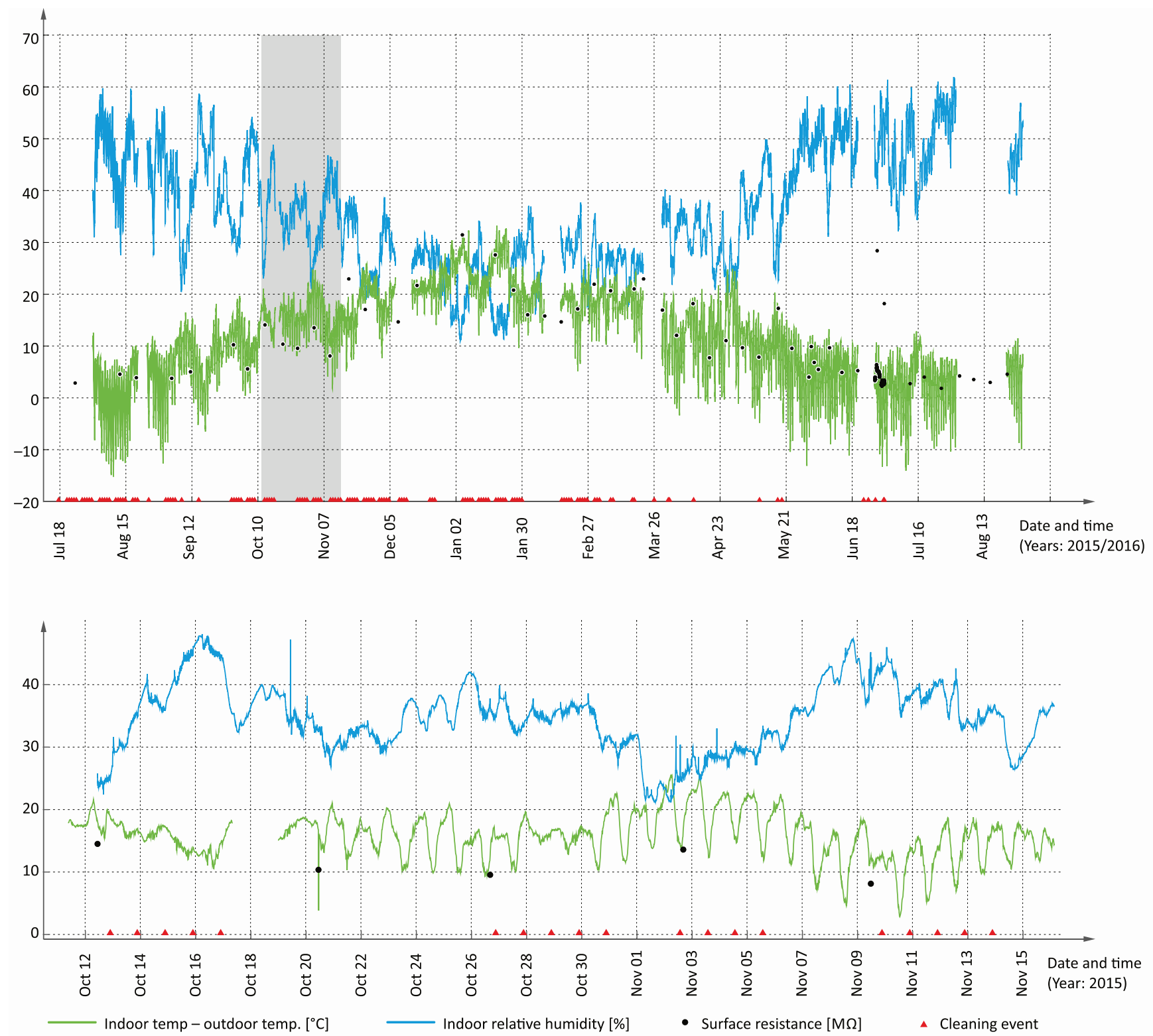

Figure 4. Side-by-side view of the difference between indoor and outdoor temperature (green), indoor relative humidity (blue), and surface resistance (black circles) throughout the entire measurement period (top), and in the interval marked by the grey background in the top graph (bottom). The triangles above the horizontal axis denote logged surface cleaning events. 
It is also worth noticing that surface resistance measurements consistently returned lower or higher values in specific regions of the worksurface - the pattern could be observed regardless of the time elapsed after the last cleaning event (Figure 5). This implies that position-dependent characteristics of surface resistance can possibly be modelled, yet, the uncertainty of the recorded measurements suggests that estimating the resistance characteristics of the entire surface based on a reduced number of measurement points will quickly encounter its limits.

\section{DATA PREPARATION AND MODELING}

\subsection{Definition of modelling assignments}

Two modelling assignments were foreseen for this R\&D project, examining (1) the dependence of floating dust density on other ambient conditions (indoor and outdoor temperature, and relative humidity) and work-dependent periodicity, and (2) the dependence of worksurface resistance on ambient conditions, cleaning events and work activity. In both cases, we looked back on pre-transformed measurement values and a fixed set of their statistical features aggregated over selected time intervals (Table 2). In order to model accumulation and saturation processes of worksurface deposits, elapsed time and floating dust density integrated since the last cleaning event were also added to the data set. In the case of dust density estimation, possible work-related periodicity was taken into account by inserting the number of the current hour, shift, workday and week (as an incremented index) into the data set. The sparse sampling of resistance values did not allow the latter indexing in the case of surface resistance modelling. In order to include position-dependent characteristics of surface resistance, the two location indices of the measurement points $(\{A \cdots E\}$, $\{1 \cdots 4\}$ in Figure 1) were added as mandatory inputs to the resistance model.

\subsection{Modelling with feature selection and neural networks}

For building the models and finding relevant dependencies, a feature selection metaheuristic method was used in combination with artificial neural networks [31].

In preparation for feature selection, the raw measurement data are augmented by a number of statistical characteristics (as in Table 2) calculated over various time windows - all these, along with the measurement data, will comprise input candidates. The reason for the large set of calculated properties is the assumption that, in such complex environments as a production facility, raw measurements alone may not fully capture all relevant dependencies or the dynamics of underlying processes. It is, then, the task of feature selection to determine which of the candidates are relevant, and which should rather be discarded as they would make the model unnecessarily large and potentially less accurate.

The feature selection method ranks the input candidates by relevance - this can be understood as a preliminary (automatic) analysis of the effect of ambient conditions and any possible hidden dynamics on the quantities regarded as output (dust density or surface resistance, in our case). The algorithm applied here is a generalisation of the method by Devijver and Kittler [32], mapping continuous parameters onto the originally discrete classification scheme with appropriate heuristics. Here, the values of the output encountered in the training data set are grouped into the highest possible number of clusters (i.e., intervals of equal length), so that at least one element is contained in each interval. Once the continuous output vector is transformed into a discrete range, the feature selection algorithm can be applied to rank the input features based on relevance. This is done by using sequential forward selection and applying a statistical measure which aims to maximize the separability of the output classes. The following equations define the statistical measure:

$M=\frac{s_{b}}{s_{w}}$,

$S_{b}=\sum_{i=1}^{c} \frac{n_{i}}{n}\left(\underline{m}_{i}-\underline{m}\right)\left(\underline{m}_{i}-\underline{m}\right)^{T}$,

$S_{w}=\sum_{i=1}^{c} \frac{n_{i}}{n} \frac{\sum_{j=1}^{n_{i}}\left(\underline{p}_{i j}-\underline{m}_{i}\right)\left(\underline{p}_{i j}-\underline{m}_{i}\right)^{T}}{n_{i}}$,

where $c$ is the number of classes of the output, $n_{i}$ is the number of samples in the $i^{\text {th }}$ class, $n$ is the number of samples, $\underline{m}_{i}$ is the centre of gravity of the $i^{t h}$ class, $\underline{m}$ is the centre of gravity of the samples and $p_{i j}$ is the $f^{\text {th }}$ sample of the $i^{\text {th }}$ class. Vector parameters $\underline{p}_{i j}, \underline{m}_{i}$ and $\underline{m}$ are defined in a subset of the whole feature set, i.e., the dimension of these vectors equals to the number of features contained in the subset. The dimension of $p_{i j}, \underline{m}_{i}$ and $\underline{m}$ is increasing over the iterations of the sequential forward selection as features are selected incrementally. In a given iteration, the newly selected feature is the one where the $M$ value of the containing subset is the highest. $S_{b}$ represents the average distance between the classes, $S_{w}$ represents the average distance within the classes, and $M$ has to be maximized in each iteration for the classes to be the most separated in a given subset. At the end of the procedure, inputs and related dependencies are ranked by relevance, allowing the number of inputs to be trimmed by omitting the least significant ones. Variable ranks determined by feature

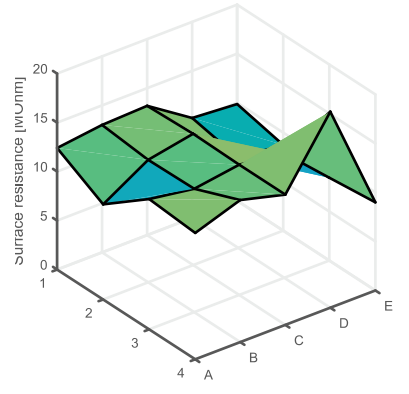

Date: $04 / 04 / 2016$, RH $30.50 \%$

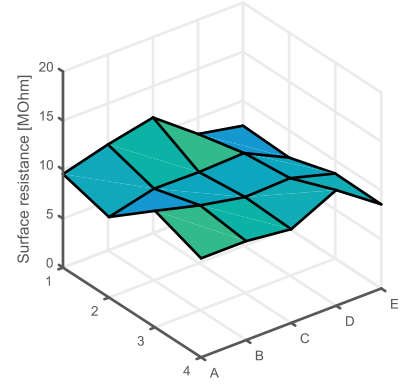

Date: 06/08/2016, RH 37.70\%

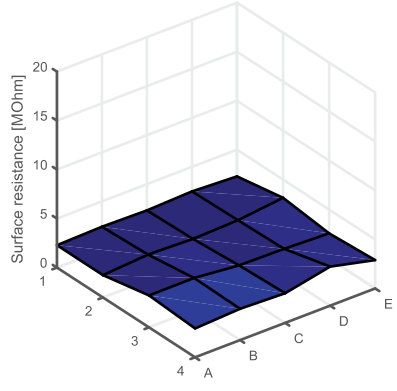

Date: $07 / 12 / 2016$, RH $52.90 \%$

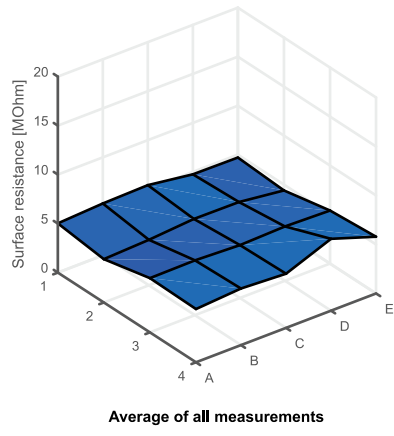

Figure 5. Surface resistance measured at selected points of the same worksurface under various ambient conditions, and average of all measurements (right) - a consistent pattern of higher/lower resistance values can be recognized. 
Table 2. List of calculated statistical properties of measured quantities used in the feature selection phase of model acquisition. Note that raw data were previously transformed to align with equidistant sampling, and derivatives could be simplified to differences as a consequence.

Calculated statistical property
No. and description

1.

1st moment

(mean, also denoted $\bar{x}$ )

2.
$\begin{aligned} & \text { nd moment } \\ & \text { (variance) }\end{aligned}$

3.

3rd moment

(skewness)

$$
\frac{\frac{\sum_{i=0}^{n}(x[i]-\bar{x})^{3}}{n}}{\left(\frac{\sum_{i=0}^{n}(x[i]-\bar{x})^{2}}{n}\right)^{\frac{3}{2}}}
$$

4.

4th moment

(kurtosis)

$$
\frac{\frac{\sum_{i=0}^{n}(x[i]-\bar{x})^{4}}{n}}{\left(\frac{\sum_{i=0}^{n}(x[i]-\bar{x})^{2}}{n}\right)^{2}}-3
$$

5.

Mean of 1st derivative

(derivative simplified to difference)

$$
\frac{\sum_{i=0}^{n} x^{\prime}[i]}{n}
$$

6.

Mean of 2nd derivative

(derivative simplified to difference)

$$
\frac{\sum_{i=0}^{n} x^{\prime \prime}[i]}{n}
$$

7. $\quad \sqrt{\frac{\frac{\sum_{i=0}^{n}\left(x^{\prime}[i]-\overline{x^{\prime}}\right)^{2}}{n}}{\frac{\sum_{i=0}^{n}(x[i]-\bar{x})^{2}}{n}}}$

8.
Complexity
$\frac{\frac{\sum_{i=0}^{n}\left(x^{\prime \prime}[i]-\overline{x^{\prime \prime}}\right)^{2}}{n}}{\frac{\sum_{i=0}^{n}\left(x^{\prime}[i]-\overline{x^{\prime}}\right)^{2}}{n}}-\frac{\frac{\sum_{i=0}^{n}\left(x^{\prime}[i]-\overline{x^{\prime}}\right)^{2}}{n}}{\frac{\sum_{i=0}^{n}(x[i]-\bar{x})^{2}}{n}}$

$$
9 .
$$

Characteristic of

autocorrelation function

$$
\frac{\sum_{i=0}^{n}(x[i]-\bar{x})\left(x^{\prime}[i+\tau]-\bar{x}\right)}{\sum_{i=0}^{n}(x[i]-\bar{x})^{2}}
$$

Minimum
min $x[i]$

Maximum
max
Mi=0 $[i]$

\section{2.}

Difference of

minimum and maximum

$$
\max _{i=0}^{n} x[i]-\min _{i=0}^{n} x[i]
$$

selection are shown for two examples: Table 3 and Figure 6 show variables and the $M$ values obtained upon their selection for estimating average dust density in the next shift, while Table 4 and Figure 7 show the same for surface resistance estimation.

After the feature selection stage, artificial neural network (ANN) models can be fitted on the first $n$ variables $\left(n_{\max }=75\right.$ or $n_{\max }=50$ in our case) to estimate the output variable. A wide range of ANN types can be used for fitting models onto the inputs prepared - however, best experience has, so far, been

Calculated statistical property $\quad$ Formula
No. and description

13.

Ratio of

minimum and maximum

$$
\frac{\max _{i=0}^{n} x[i]}{\min _{i=0}^{n} x[i]}
$$

14. Total count of quantity crossing its $\quad \sum_{\operatorname{sgn}(x[i]+\bar{x}) \neq \operatorname{sgn}(x[i+1]+\bar{x})} 1$
mean in any direction

15.

$\begin{array}{ll}\text { First occurrence of quantity } & \min _{x[i]>\bar{x}} x[i] \\ \text { becoming greater than its mean } & \end{array}$

16.

Last occurrencece of quantity $\quad \max _{x[i]>\bar{x}} x[i]$

becoming greater than its mean

17.

Total count of samples when $\quad \sum_{x[i]>\bar{x}} 1$

quantity is larger than its mean

$\sum_{x[i]<\bar{x}} 1$

Total count of samples when

quantity is smaller than its mean

19.

Total count of samples when

quantity is greater than both

$\left.\sum_{(x[i]>x[i-1]) \wedge(x[i]>x[i+1])}\right)^{1}$

neighbouring samples

20.

Total count of samples when

quantity is smaller than both

neighbouring samples

$\sum_{(x[i]<x[i-1]) \wedge(x[i]<x[i+1])} 1$

21.

Value of first sample

$x[0]$

22.

Value of last sample

$x[n]$

23.

Difference of last and first sample

$x[n]-x[0]$

24.

Ratio of last and first sample

$\frac{x[n]}{x[0]}$

made with simple Multilayer Perceptron (MLP) networks. In the cases described here, the networks had $n$ inputs (depending on the specific modelling task), one output, and one hidden layer with 8 neurons. The dimensioning of the hidden layer relied on empirical experience with previous industrial modelling tasks of comparable complexity [31]. Data sets were assembled from the $n$ selected inputs and the corresponding output, and training, validation and test sets were selected by random choice from the assembled vectors [33]. Training was performed with variable-rate gradient descent, using the Levenberg-Marquardt 
Table 3. Ranking of the first 50 calculated statistical properties of measured quantities and their calculation windows for next-shift estimation of dust density (see equations (1) - (3) for the definition of the measure $M$, and Table 2 for the definition of statistical properties).

\begin{tabular}{|c|c|c|c|c|}
\hline $\begin{array}{l}\text { Feature } \\
\text { No. }\end{array}$ & Measured quantity & $\begin{array}{l}\text { Calculated statistical } \\
\text { property No. }\end{array}$ & $\begin{array}{l}\text { Interval observed for } \\
\text { calculation of property }\end{array}$ & $\begin{array}{l}\text { Feature selection } \\
\text { measure (M) }\end{array}$ \\
\hline 1 & Dust density, vicinity of workstation & 1 & Preceding 3 days & 7.6254 \\
\hline 2 & Dust density, vicinity of workstation & 1 & Preceding 2 shifts & 7.5924 \\
\hline 3 & Dust density, vicinity of workstation & 10 & Preceding 2 shifts & 7.2220 \\
\hline 4 & Dust density, vicinity of workstation & 1 & Preceding 7 days & 6.8240 \\
\hline 5 & Dust density, vicinity of workstation & 1 & Preceding 3 shifts & 6.4547 \\
\hline 6 & Dust density, vicinity of workstation & 10 & Preceding 3 shifts & 6.1757 \\
\hline 7 & Dust density, vicinity of workstation & 7 & Preceding 7 days & 5.9740 \\
\hline 8 & Dust density, vicinity of workstation & 7 & Preceding 3 days & 5.7800 \\
\hline 9 & Outdoor temperature, measured at HVAC rooftop unit 4 & 13 & Preceding 3 shifts & 5.6091 \\
\hline 10 & Dust density, vicinity of workstation & 10 & Preceding 3 days & 5.4629 \\
\hline 11 & Outdoor temperature, measured at HVAC rooftop unit 6 & 13 & Preceding 3 shifts & 5.3280 \\
\hline 12 & Outdoor temperature, measured at HVAC rooftop unit 4 & 13 & Preceding 2 shifts & 5.2032 \\
\hline 13 & Outdoor temperature, measured at HVAC rooftop unit 6 & 13 & Preceding 2 shifts & 5.0767 \\
\hline 14 & Relative humidity measured manually at workstation & 1 & Preceding 3 days & 4.9283 \\
\hline 15 & Relative humidity measured manually at workstation & 10 & Preceding 3 days & 4.8119 \\
\hline 16 & Relative humidity measured manually at workstation & 11 & Preceding 3 days & 4.7242 \\
\hline 17 & Dust density, vicinity of workstation & 10 & Preceding 7 days & 4.6466 \\
\hline 18 & Relative humidity, vicinity of workstation & 19 & Preceding 2 shifts & 4.5691 \\
\hline 19 & Relative humidity, vicinity of workstation & 20 & Preceding 2 shifts & 4.4847 \\
\hline 20 & Outdoor temperature, measured at HVAC rooftop unit 4 & 24 & Preceding 7 days & 4.4021 \\
\hline 21 & Outdoor temperature, measured at HVAC rooftop unit 6 & 24 & Preceding 7 days & 4.3245 \\
\hline 22 & Outdoor temperature, measured at HVAC rooftop unit 4 & 9 & Preceding 3 shifts & 4.2368 \\
\hline 23 & Outdoor temperature, measured at HVAC rooftop unit 6 & 9 & Preceding 3 shifts & 4.1576 \\
\hline 24 & Indoor temperature, measured at HVAC rooftop unit 4 & 9 & Preceding 3 shifts & 4.0749 \\
\hline 25 & Wet bulb temperature, vicinity of workstation & 20 & Preceding 3 shifts & 3.9990 \\
\hline 26 & Indoor relative humidity, measured at HVAC rooftop unit 6 & 9 & Preceding 3 shifts & 3.9248 \\
\hline 27 & Dust density, vicinity of workstation & 7 & Preceding 2 shifts & 3.8506 \\
\hline 28 & Fan air temperature, measured at HVAC rooftop unit 4 & 9 & Preceding 2 shifts & 3.7849 \\
\hline 29 & Relative humidity, vicinity of workstation & 19 & Preceding 3 shifts & 3.7241 \\
\hline 30 & Outdoor temperature, measured at HVAC rooftop unit 4 & 9 & Preceding 2 shifts & 3.6567 \\
\hline 31 & Outdoor temperature, measured at HVAC rooftop unit 6 & 9 & Preceding 2 shifts & 3.5977 \\
\hline 32 & Dust density, vicinity of workstation & 7 & Preceding 3 shifts & 3.5403 \\
\hline 33 & Wet bulb temperature, vicinity of workstation & 20 & Preceding 2 shifts & 3.4830 \\
\hline 34 & Outdoor temperature, measured at HVAC rooftop unit 4 & 24 & Preceding 3 days & 3.4307 \\
\hline 35 & Dust density, vicinity of workstation & 13 & Preceding 7 days & 3.3835 \\
\hline 36 & Outdoor temperature, measured at HVAC rooftop unit 6 & 24 & Preceding 3 days & 3.3295 \\
\hline 37 & Fan air temperature, measured at HVAC rooftop unit 6 & 9 & Preceding 3 shifts & 3.2823 \\
\hline 38 & Indoor temperature, measured at HVAC rooftop unit 6 & 2 & Preceding 2 shifts & 3.2367 \\
\hline 39 & Indoor temperature, measured at HVAC rooftop unit 6 & 2 & Preceding 3 shifts & 3.1929 \\
\hline 40 & Indoor temperature, measured at HVAC rooftop unit 6 & 9 & Preceding 3 shifts & 3.1490 \\
\hline 41 & Indoor relative humidity, measured at HVAC rooftop unit 4 & 9 & Preceding 3 shifts & 3.1086 \\
\hline 42 & Indoor relative humidity, measured at HVAC rooftop unit 6 & 9 & Preceding 2 shifts & 3.0597 \\
\hline 43 & Fan air temperature, measured at HVAC rooftop unit 6 & 9 & Preceding 2 shifts & 3.0259 \\
\hline 44 & Indoor temperature, measured at HVAC rooftop unit 6 & 12 & Preceding 2 shifts & 2.9770 \\
\hline 45 & Indoor temperature, measured at HVAC rooftop unit 6 & 9 & Preceding 2 shifts & 2.9365 \\
\hline 46 & Indoor temperature, measured at HVAC rooftop unit 6 & 12 & Preceding 3 shifts & 2.8961 \\
\hline 47 & Indoor relative humidity, measured at HVAC rooftop unit 4 & 9 & Preceding 2 shifts & 2.8556 \\
\hline 48 & Indoor temperature, measured at HVAC rooftop unit 6 & 10 & Preceding 2 shifts & 2.8168 \\
\hline 49 & Outdoor temperature, measured at HVAC rooftop unit 4 & 24 & Preceding 3 shifts & 2.7848 \\
\hline 50 & Outdoor temperature, measured at HVAC rooftop unit 6 & 24 & Preceding 3 shifts & 2.7409 \\
\hline
\end{tabular}

algorithm [34]. The resulting models can be ordered by increasing number of inputs, and the model error can be observed to see how many input variables are necessary to reach a point where the model error cannot be lowered significantly by adding more of the input variables ranked by feature selection. Figures 8 and 9 show the graph of mean model errors in dependence of the number of input variables used for model building. 
Table 4. Ranking of the first 50 calculated statistical properties of measured quantities and their calculation windows for estimation of current surface resistance. Note that, as opposed to the usual behaviour, $M$ has a low initial value which rises gradually. Forced input variables placed in the first rank(s) are known to have such effect - this, however, does not compromise subsequent ranking by calculated $M$ values.

\begin{tabular}{|c|c|c|c|c|}
\hline $\begin{array}{l}\text { Feature } \\
\text { No. }\end{array}$ & Measured quantity & $\begin{array}{l}\text { Calculated statistical } \\
\text { property No. }\end{array}$ & $\begin{array}{l}\text { Interval observed for } \\
\text { calculation of property }\end{array}$ & $\begin{array}{l}\text { Feature selection } \\
\text { measure (M) }\end{array}$ \\
\hline 1 & Position of measuring point on worksurface & N/A & N/A & $\mathrm{N} / \mathrm{A}$ \\
\hline 2 & Relative humidity measured manually at workstation & 1 & Preceding 7 days & 0.8199 \\
\hline 3 & Relative humidity measured manually at workstation & 10 & Preceding 7 days & 1.3981 \\
\hline 4 & Relative humidity measured manually at workstation & 11 & Preceding 7 days & 2.0236 \\
\hline 5 & Relative humidity measured manually at workstation & 21 & Preceding 7 days & 2.5295 \\
\hline 6 & Relative humidity measured manually at workstation & 22 & Preceding 7 days & 2.9303 \\
\hline 7 & Outdoor temperature, measured at HVAC rooftop unit 6 & 1 & Preceding 7 days & 3.1420 \\
\hline 8 & Dust density, vicinity of workstation & 10 & Preceding 3 days & 3.3183 \\
\hline 9 & Dust density, vicinity of workstation & 5 & Preceding 7 days & 3.4601 \\
\hline 10 & Dust density, vicinity of workstation & 8 & Preceding 3 days & 3.5848 \\
\hline 11 & Dust density, vicinity of workstation & 6 & Preceding 7 days & 3.6992 \\
\hline 12 & Outdoor temperature, measured at HVAC rooftop unit 6 & 11 & Preceding 7 days & 3.7867 \\
\hline 13 & Dust density, vicinity of workstation & 5 & Preceding 3 days & 3.8561 \\
\hline 14 & Dust density, vicinity of workstation & 10 & Preceding 7 days & 3.9137 \\
\hline 15 & Dust density, vicinity of workstation & 6 & Preceding 3 days & 3.9633 \\
\hline 16 & Dust density, vicinity of workstation & 8 & Preceding 3 days & 4.0053 \\
\hline 17 & Outdoor temperature, measured at HVAC rooftop unit 4 & 1 & Preceding 7 days & 4.0444 \\
\hline 18 & Dust density, vicinity of workstation & 1 & Preceding 7 days & 4.0673 \\
\hline 19 & Outdoor temperature, measured at HVAC rooftop unit 6 & 1 & Preceding 3 days & 4.0789 \\
\hline 20 & Dust density, vicinity of workstation & 7 & Preceding 7 days & 4.0940 \\
\hline 21 & Indoor relative humidity, measured at HVAC rooftop unit 6 & 1 & Preceding 7 days & 4.1084 \\
\hline 22 & Dust density, vicinity of workstation & 10 & Preceding 1 shift & 4.1240 \\
\hline 23 & Dust density, vicinity of workstation & 13 & Preceding 3 days & 4.1409 \\
\hline 24 & Indoor relative humidity, measured at HVAC rooftop unit 6 & 21 & Preceding 3 days & 4.1515 \\
\hline 25 & Dust density, vicinity of workstation & 1 & Current shift & 4.1588 \\
\hline 26 & Dust density, vicinity of workstation & 1 & Current hour & 4.1637 \\
\hline 27 & Dust density, vicinity of workstation & 7 & Current shift & 4.1668 \\
\hline 28 & Dust density, vicinity of workstation & 10 & Current shift & 4.1685 \\
\hline 29 & Dust density, vicinity of workstation & 7 & Current hour & 4.1695 \\
\hline 30 & Dust density, vicinity of workstation & 10 & Current hour & 4.1707 \\
\hline 31 & Outdoor temperature, measured at HVAC rooftop unit 4 & 1 & Preceding 3 days & 4.1717 \\
\hline 32 & Dust density, vicinity of workstation & 1 & Preceding 1 hour & 4.1728 \\
\hline 33 & Dust density integrated since last cleaning & 19 & Preceding 1 hour & 4.1741 \\
\hline 34 & Dust density integrated since last cleaning & 20 & Preceding 1 hour & 4.1748 \\
\hline 35 & Dust density integrated since last cleaning & 19 & Preceding 3 shifts & 4.1751 \\
\hline 36 & Dust density integrated since last cleaning & 20 & Preceding 3 shifts & 4.1756 \\
\hline 37 & Relative humidity measured manually at workstation & 2 & Preceding 7 days & 4.1761 \\
\hline 38 & Relative humidity measured manually at workstation & 12 & Preceding 7 days & 4.1771 \\
\hline 39 & Relative humidity measured manually at workstation & 13 & Preceding 7 days & 4.1780 \\
\hline 40 & Relative humidity measured manually at workstation & 23 & Preceding 7 days & 4.1789 \\
\hline 41 & Relative humidity measured manually at workstation & 24 & Preceding 7 days & 4.1800 \\
\hline 42 & Temperature measured manually at workstation & 2 & Preceding 7 days & 4.1808 \\
\hline 43 & Temperature measured manually at workstation & 12 & Preceding 7 days & 4.1823 \\
\hline 44 & Temperature measured manually at workstation & 13 & Preceding 7 days & 4.1832 \\
\hline 45 & Temperature measured manually at workstation & 23 & Preceding 7 days & 4.1843 \\
\hline 46 & Temperature measured manually at workstation & 24 & Preceding 7 days & 4.1851 \\
\hline 47 & Dust density integrated since last cleaning & 2 & Preceding 1 hour & 4.1859 \\
\hline 48 & Indoor temperature, measured at HVAC rooftop unit 6 & 2 & Current hour & 4.1864 \\
\hline 49 & Indoor temperature, measured at HVAC rooftop unit 6 & 2 & Preceding 1 hour & 4.1868 \\
\hline 50 & Outdoor temperature, measured at HVAC rooftop unit 4 & 6 & Preceding 1 hour & 4.1869 \\
\hline
\end{tabular}

Note that for each number of selected - most relevant inputs, a new network was trained, and the mean relative error of the trained network is shown in the graphs in Figures 8 and
9, and not, as typical, the mean error of the same network during a training procedure. 


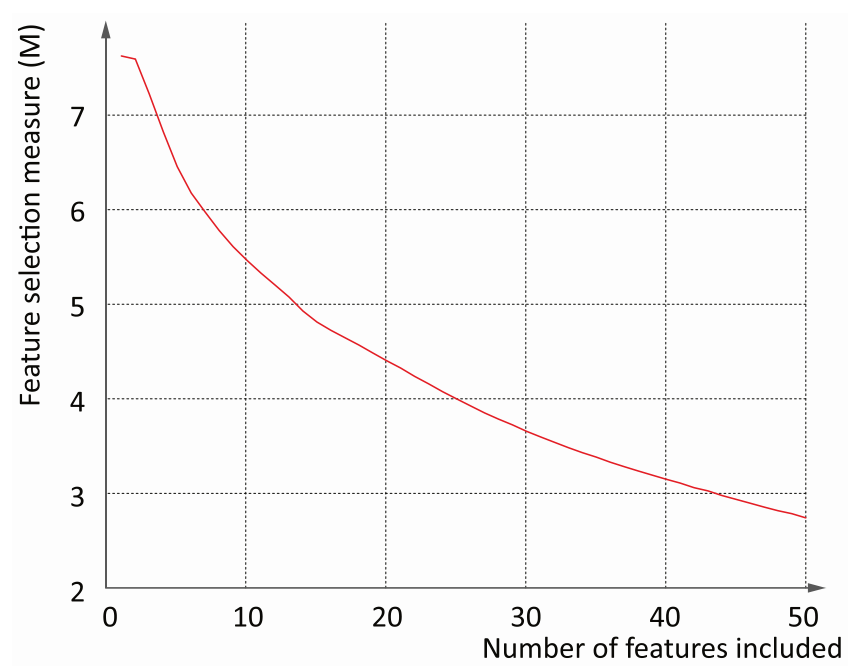

Figure 6. Feature selection measure of input candidates ranked for shift-byshift estimation of floating dust density (see also Table 3 for detailed data).

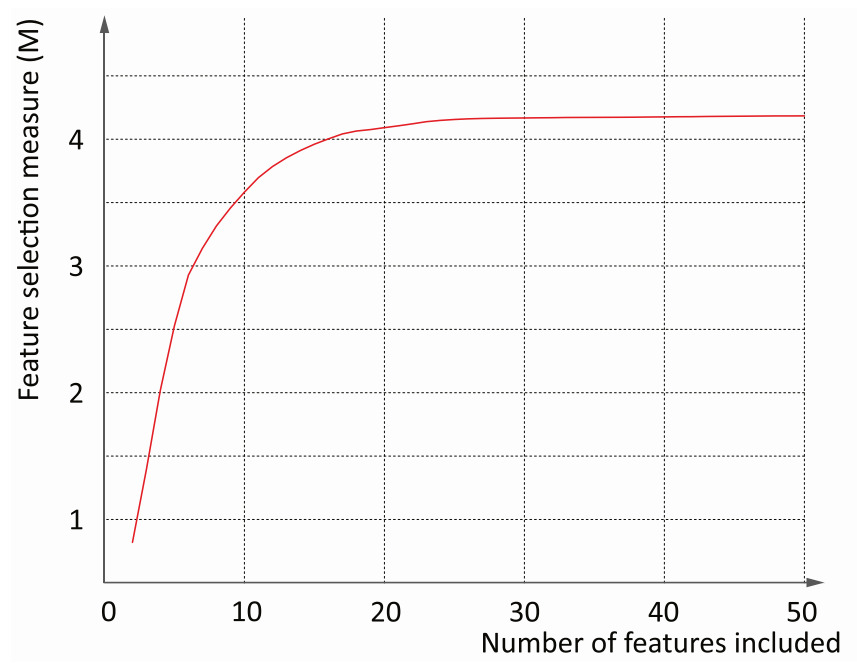

Figure 7. Feature selection measure of input candidates ranked for estimation of surface resistance (see also Table 4 for detailed data).

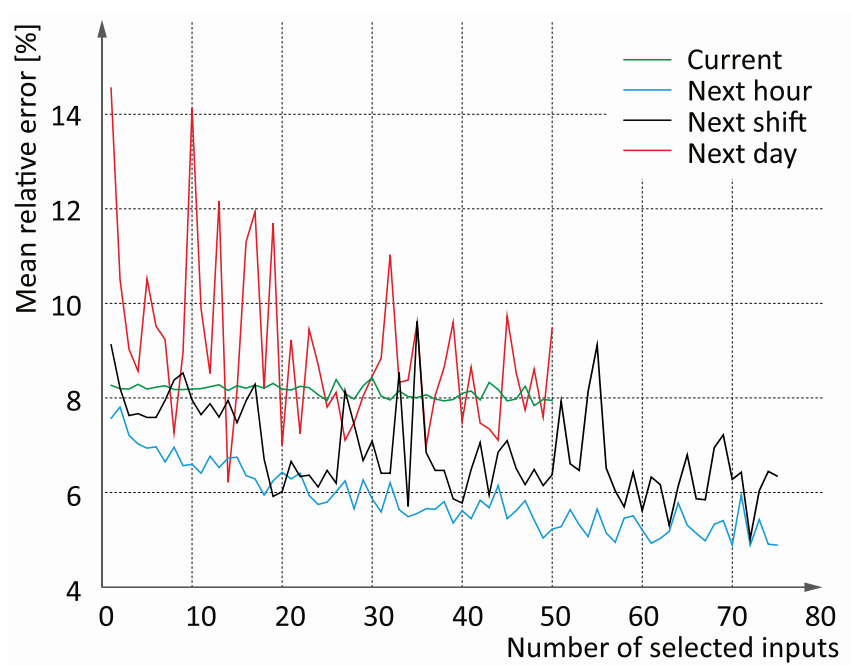

Figure 8. Error rates of models estimating dust density for different time windows, in dependence of the number of most relevant input variables selected.

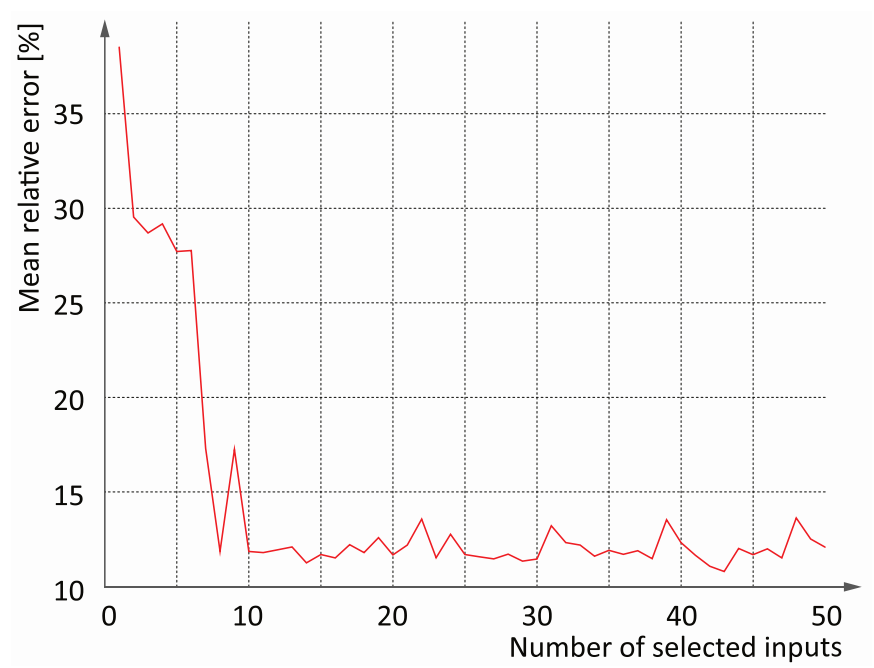

Figure 9. Error rate of the model estimating worksurface resistance, in dependence of the number of most relevant input variables selected.

\subsection{Modelling solutions and their evaluation}

ANN models for floating dust density estimations were prepared for current value, and prediction windows of the next hour, next shift, and next day. Average error rates of 5-8\% were attained with the 50 best input variables included in the ANN, and reasonably close results ( $6-8 \%$ error) were reached with the most relevant 10-15 inputs (see also Table 5). For all of the dust density estimations, statistical features of previous dust densities were found most relevant, typically in the range of some shifts or days prior to estimation (see also Table 3). Remarkable was also the presence of outdoor temperature values among the most relevant variables - at this point, this is assumed to be the effect of increased fan air stream in the air conditioned inner space when indoor and outdoor temperatures differ largely. In case the dependence on fan air stream holds, facility-wide dust density measurements are recommended, as dust is known to exhibit uneven distribution and settling patterns in the presence of air streams, especially if the latter are themselves potential dust sources.

The ANN model estimating surface resistance (current values only) showed a mean relative error below $12 \%$ already after including the 10 most relevant inputs, and did not improve much thereafter (Table 6). In the feature selection round, the relative humidity values from the past 7 days were found to be of highest relevance, followed by outdoor temperature and dust density of the preceding 3-7 days (Table 3). As mentioned before, long-lasting low outdoor temperatures are known to deplete humidity of heated indoor spaces, and were found to have effect on dust density as well via increased fan air stream. Interestingly, effects of cleaning events were ranked $33^{\text {rd }}$ and behind, possibly implying that resistance measurements were carried out too sparsely to capture their influence.

\section{NOVELTIES AND CONCLUSIONS}

The paper presented first results of an $R \& D$ project in an area of industrial production that has rarely been in the focus of research, namely, the dependence of the surface resistance of ESD-protected worksurfaces on ambient conditions and work processes in an electronics assembly and repair context. An 
Table 5. Mean relative error of dust density estimations for various estimation intervals and numbers of features.

\begin{tabular}{|c|c|c|c|c|}
\hline Interval of estimation & $\begin{array}{l}\text { Relative error } \\
\text { for first } 10 \text { features }\end{array}$ & $\begin{array}{l}\text { Relative error } \\
\text { for first } 15 \text { features }\end{array}$ & $\begin{array}{l}\text { Relative error } \\
\text { for first } 20 \text { features }\end{array}$ & $\begin{array}{l}\text { Minimal relative error } \\
\text { (number of features) }\end{array}$ \\
\hline Current & $8.19 \%$ & $8.26 \%$ & $8.19 \%$ & $7.84 \%$ (48 features) \\
\hline Next hour & $6.60 \%$ & $6.75 \%$ & $6.43 \%$ & $7.89 \%$ (70 features) \\
\hline Next shift & $7.96 \%$ & $7.48 \%$ & $6.02 \%$ & $5.03 \%$ (73 features) \\
\hline Next day & $14.13 \%$ & $8.17 \%$ & $7.01 \%$ & $6.22 \%$ (14 features) \\
\hline
\end{tabular}

Table 6. Mean relative error of surface resistance estimations for various numbers of features.

\begin{tabular}{|l|l|l|l|l|l|l}
\hline $\begin{array}{l}\text { Relative error } \\
\text { for first } \mathbf{1 0} \text { features }\end{array}$ & $\begin{array}{l}\text { Relative error } \\
\text { for first 15 features }\end{array}$ & $\begin{array}{l}\text { Relative error } \\
\text { for first 20 features }\end{array}$ & $\begin{array}{l}\text { Minimal relative error } \\
\text { (number of features) }\end{array}$ \\
\hline $11.87 \%$ & $11.71 \%$ & $11.69 \%$ & $10.81 \%(43$ features) \\
\hline
\end{tabular}

important characteristic of the research presented is its closeness to practical application - (1) existing industrial experience played a key role in outlining expected dependencies and setting up an instrumentation roadmap, and (2) results continue to be evaluated in the context of a possible roll-out in industrial production where measuring and intervention must align well with efficient manufacturing routine. It is also important to stress that measurements were carried out in a live industrial environment where ambient conditions cannot be varied at will, and measurements cannot impair ongoing work processes. While such conditions differ largely from laboratory tests, they are often encountered during improvement or retrofit of existing production facilities, especially if the subject of investigation cannot be cleanly separated from a complex network of interdependencies of the manufacturing environment.

Measured data of ambient conditions and surface resistance were examined by a feature selection method, revealing that surveying the ambient conditions for the preceding 3-7 days allows a resistance estimation with $12 \%$ relative error without relying on resistance measurement records from these intervals. This allows surface resistance estimation with sensors that do not interfere with ongoing work processes, although with limited accuracy. While these results alone already show that a model-based estimation tool is feasible, relevance rankings of cleaning times suggest that a more accurate model is likely to need more frequent resistance measurement, at least in the data collection phase.

The results presented in the paper focused on one selected workstation where the material of the ESD-protected worksurface was not changed during the measurements. The examined workstation was equipped with good quality protective materials, and critically low humidity values were never experienced during the data collection interval therefore, extension of the measurements to inferior protective materials is recommended in order to gain more insight into differences to be expected at other production sites. Also, measurements would have to be taken over an extended timespan to capture gradual changes, e.g., due to material aging, or variations in worksurface contaminants due to major changes in the product mix, tooling, etc. Compliance with production processes has not allowed so far to leave the safe area of ambient parameters - follow-up research will have to include this option for better examination of the boundaries of safe work process conditions.
While the potential relevance of optical (contactless) surface contamination measurement in ease of use and minimal impact on work activities was highlighted in the paper, limitations of the current project will leave it for later examination. Infrared imaging is all the more interesting if the high fluctuation of resistance measurements is in correlation with local differences of infrared reflectivity of the surface.

Research presented in the paper was carried out by industrial incentive, and, consequently, had to adapt to the conditions and preferences of a given manufacturing context. While budget and workforce limitations have not allowed research to step far beyond the aforementioned scope, the sparse coverage of surface resistance models in literature suggests that follow-up research will provide more insight regarding underlying theoretical models as well as application in an industrial environment. In this regard, laboratory measurements, isolated from the complex interdependencies of field deployment, as well as more comprehensive testing of various model building approaches are expected convey an added value in the application context of the electronics manufacturing industry.

\section{ACKNOWLEDGEMENT}

Work presented here has been supported by the grants of the Highly Industrialised Region in Western Hungary with limited R\&D capacity: "Strengthening of the regional research competencies related to future-oriented manufacturing technologies and products of strategic industries by a research and development program carried out in comprehensive collaboration”, under grant No. VKSZ_12-1-2013-0038.

\section{REFERENCES}

[1] R. B. Rosner, "Conductive materials for ESD applications: an overview", IEEE Transactions on Device and Materials Reliability, 1 (2001) pp. 9-16.

[2] ESD Association, Working Group 4, "Survey of worksurfaces and grounding mechanisms (ESD TR4.0-01-02)”, 2015.

[3] T. Sugimoto, M. Abe, Y. Higashiyama, "Noncontact surface resistivity measurement using a cylindrical surface potential detector with a corona charger", IEEE Transactions on Industry Applications, 48 (5) (2012) pp. 1657-1661.

[4] Zs. Kemény, Zs. J. Viharos, K. B. Kis, R. Csontos, T. Kovács, K. Németh, "Measurement and estimation of surface resistance on ESD-protected workstations", Proc. of the 21st IMEKO TC4 
International Symposium on Understanding the World through Electrical and Electronic Measurement (2016), pp. 208-213.

[5] J. Davidson, T. Williams, A. Bailey, G. Hearn, "Characterisation of electrostatic discharges from insulating surfaces", Journal of Electrostatics, 51 (2001) pp. 374-380.

[6] H. Gossner, K. Esmark, W. Stadler, Simulation methods for ESD protection development, Elsevier, 2003.

[7] ESD Association, "ESDA-JEDEC joint standard for electrostatic discharge sensitivity testing: Human body model (HBM), component level (ANSI-ESDAJEDEC- JS-001-2014)", 2014.

[8] ESD Association, "ESDA standard test method for electrostatic discharge sensitivity testing: Machine model (MM), component level (ANSI-ESD-STM5.2-2012)", 2013.

[9] G. Kietzer, "ESD risks in the electronics manufacturing", Proc. of the 34th Electrical Overstress/- Electrostatic Discharge Symposium (EOS/ESD), 2012 pp. 1-8.

[10] A. Morando, M. Borsero, A. Sardi, G. Vizio, "Modeling and practical suggestions to improve ESD immunity test repeatability", Proc. 17th Symposium IMEKO TC 4, 2010 pp. $563-567$.

[11] L. Toma, A. Salceanu, M. Cretu, "ESD immunity tests in system designs", Proc. 15th Symposium IMEKO TC 4, 2007 pp. 1-4.

[12] S. P. Singh, H. El-Khateeb, "Evaluation of a proposed test method to measure surface and volume resistance of static dissipative packaging materials", Packaging Technology and Science, 7 (6) (1994) pp. 283-289.

[13] ESD Association, "ESDA standard for the protection of electrostatic discharge susceptible items-static control bags (ANSI-ESD S11.4-2012)", 2012.

[14] ESD Association, "ESDA standard for the protection of electrostatic discharge susceptible items-packaging materials for ESD-sensitive items (ANSIESD S541-2008)", 2008.

[15] A. Hebeish, M. El-Gamal, T. Said, R. A. El-Hady, "Major factors affecting the performance of ESD-protective fabrics", The Journal of the Textile Institute, 101 (5) (2010) pp. 389-398.

[16] J. Brodbeck, B. Grunden, "Humidity effects on laminated ESD worksurface resistance and charge dissipation properties", Proc. of the 26th Electrical Overstress/- Electrostatic Discharge Symposium (EOS/ESD), 2004.

[17] D. Swenson, J. Weidendorf, D. Parkin, E. Gillard, "Resistance to ground and tribocharging of personnel, as influenced by relative humidity", Proc. of the 17th Electrical Overstress/Electrostatic Discharge Symposium (EOS/ESD), 1995.

[18] D. Blinde, L. Lavoie, "Quantitative effects of relative and absolute humidity on ESD generation/supression", Proc. of the 3rd Electrical Overstress/- Electrostatic Discharge Symposium (EOS/ESD), 1981.

[19] B. Song, M. H. Azarian, M. G. Pecht, "Effect of temperature and relative humidity on the impedance degradation of dustcontaminated electronics", Journal of The Electrochemical Society, 160 (3) (2013) pp. C97-C105.
[20] S. Sidthik, L. Kalaivani, M. W. Iruthayarajan, "Evaluation and prediction of contamination level in coastal region insulators based on leakage current characteristics", 2013 IEEE International Conference on Circuits, Power and Computing Technologies (ICCPCT), 2013 pp. 132-137.

[21] J. Volkwein, A. Maynard, M. Harper, Workplace aerosol measurement, John Wiley \& Sons, 2011.

[22] M. Kauhaniemi, J. Kukkonen, J. Härkönen, J. Nikmo, L. Kangas, G. Omstedt, M. Ketzel, A. Kousa, M. Haakana, A. Karppinen, "Evaluation of a road dust suspension model for predicting the concentrations of pm 10 in a street canyon", Atmospheric Environment, 45 (22) (2011) pp. 3646-3654.

[23] W. Kunkel, "The static electrification of dust particles on dispersion into a cloud", Journal of Applied Physics, 21 (8) (1950) pp. 820-832.

[24] H. Qasem, T. Betts, R. Gottschalg, "Spatially-resolved modelling of dust effect on cadmium telluride photovoltaic modules", Solar Energy, 90 (2013) pp. 154-163.

[25] J. Li, C. Sun, W. Sima, Q. Yang, J. Hu, "Contamination level prediction of insulators based on the characteristics of leakage current", IEEE Transactions on Power Delivery, 25 (1) (2010) pp. 417-424.

[26] B. Song, Impact of dust on the reliability of printed circuit assemblies, Ph.D. thesis, University of Maryland, 2013.

[27] A. Chudnovsky, E. Ben-Dor, "Application of visible, nearinfrared, and short-wave infrared (400-2500 $\mathrm{nm}$ ) reflectance spectroscopy in quantitatively assessing settled dust in the indoor environment. Case study in dwellings and office environments", Science of the total environment, 393 (2) (2008) pp. 198-213.

[28] Sharp Co., "GP2Y1010AU0F Compact Optical Dust Sensor", Datasheet No. E4-A01501EN, 2006.

[29] W. Warmbier, "Metriso 2000 ESD Test Instrument Operating Instructions", Technical documentation No. 3-349-087-53 (2001)

[30] ESD Association, "ESDA standard test method for the protection of electrostatic discharge susceptible items-surface resistance measurement of static dissipative planar materials (ANSI/ESD STM11.11- 2006)", 2006.

[31] Zs. J. Viharos, "Automatic generation a net of models for high and low levels of production control", IFAC Proceedings Volumes, 38 (1) (2005) pp. 159-164.

[32] P. A. Devijver, J. Kittler, "Pattern recognition, A Statistical Approach", Prentice-Hall International Inc., England, London, 1982.

[33] M. H. Beale, M. T. Hagan, H. B. Demuth, "MATLAB Neural Network Toolbox, User's Guide", The MathWorks, 2017.

[34] C. T. Kelley, "Iterative Methods for Optimization", SIAM Frontiers in Applied Mathematics, 18 (1999), ISBN 0-89871433-8, pp. 56-58. 